The impact of ocean data assimilation on seasonal-to-interannual forecasts:

A case study of the 2006 El Niño event

Shu-Chih Yang ${ }^{1,2}$, Michele Rienecker ${ }^{1}$, and Christian Keppenne ${ }^{1,3}$

${ }^{1}$ Global Modeling and Assimilation Office, NASA/GSFC, Greenbelt

${ }^{2}$ Department of Atmospheric Sciences, National Central University, Jhongli

${ }^{3}$ SAIC, Inc

\begin{abstract}
This study investigates the impact of four different ocean analyses on coupled forecasts of the 2006 El Niño event. Forecasts initialized in June 2006 using ocean analyses from an assimilation that uses flow-dependent background error covariances are compared with those using static error covariances that are not flow dependent. The flowdependent error covariances reflect the error structures related to the background ENSO instability and are generated by the coupled breeding method.

The ocean analyses used in this study result from the assimilation of temperature and salinity, with the salinity data available from Argo floats. Of the analyses, the one using information from the coupled bred vectors (BV) replicates the observed equatorial long wave propagation best and exhibits more warming features leading to the $2006 \mathrm{El}$ Niño event.

The forecasts initialized from the BV-based analysis agree best with the observations in terms of the growth of the warm anomaly through two warming phases. This better performance is related to the impact of the salinity analysis on the state evolution in the equatorial thermocline. The early warming is traced back to salinity differences in the upper ocean of the equatorial central Pacific, while the second warming, corresponding to the mature phase, is associated with the effect of the salinity assimilation on the depth of the thermocline in the western equatorial Pacific. The series of forecast experiments conducted here show that the structure of the salinity in the initial conditions is important to the forecasts of the extension of the warm pool and the evolution of the 2006 El Niño event.
\end{abstract}

\title{
Popular Summary
}

The El Niño-Southern oscillation (ENSO) is a coupled atmosphere-ocean phenomenon that appears in a form of large-scale anomalous warming or cooling in the sea surface temperature in the tropical Pacific on 3-7 year time scales. The associated atmospheric response is apparent in changes to precipitation or the likelihood of severe weather events and has a global societal impact.

The skill of ENSO prediction is related to not only the physics of the atmosphere-ocean 
coupled model but also the quality of initial conditions used to initialize the coupled model. For coupled forecasting in operational setting, the atmospheric initial states are generated by the AGCM simulation forced by realistic sea surface temperature; the ocean initial states are ocean analysis product by assimilating the ocean observations, like temperature, and salinity. Whether the initial conditions describe proper information about the ENSO instability will affect the skill to predict the ENSO event.

In the procedure of data assimilation, the background error statistics (the background error covariance) determines how to arrange the analysis correction to correct the background state, given that the observation has inhomogeneous distribution. The purpose of this study is to demonstrate how the error structures used in ocean data assimilation can modify the characteristics of seasonal-to-interannual variability in the analyses and thus have impact on the skill of ENSO prediction. The ocean analysis products are derived with different characteristics of background error covariances, given the same temperature and salinity observations during the year 2006, especially with the availability of salinity data from the Argo floats. The characteristics associated with the $2006 \mathrm{El}$ Niño event are examined for the analyses and then for the skill of coupled forecasting after the coupled GCM initialized with these analyses. The 2006 El Niño event is a mild event characterized by rapid developing and decaying stages. Most of the operations centers issued the warm prediction until the warming has emerged. Result from this study suggests that the warm anomaly could be predicted at the early developing stage when the ocean analysis is derived with a background error covariance sensitive to the background seasonal-to-interannual variations.

Among the ocean analyses used in this study, only one of them uses the error structure related to the seasonal-to-interannual uncertainties, which is provided by the ocean component of the coupled bred vectors (BVs). The BVs attempt to capture the forecast uncertainties associated with slowly varying coupled instabilities relevant to ENSO. Of the analyses used in this study, the one using information from the coupled BV replicates the observed equatorial long wave propagation best and exhibits more warming features leading to the $2006 \mathrm{El}$ Niño event. Based on the differences among the analyses and with the same atmospheric initial conditions, the forecasts initialized from the BV-based analysis agree best with the observations in terms of the growth of the warm anomaly through two warming phases. The early warming is traced back to salinity differences in the upper ocean of the equatorial central Pacific, while the second warming, corresponding to the mature phase, is associated with the effect of the salinity assimilation on the depth of the thermocline in the western equatorial Pacific.

The series of forecasts experiments conducted in this study show that the structure of the salinity and its relationship to temperature in the initial conditions are key factors in forecasts of the extension of the warm pool and the evolution of the 2006 El Nino event. 